\title{
Addendum
}

\section{p-Chlorophenylalanine-Induced Alterations in the Behavioral Effects of 5-Hydroxytryptophan}

\author{
W.O. Boggan, D.X. Freedman, and J.B. Appel \\ Psychopharmacologia (Berl.) 33, 293-298 (1973)
}

Both on the cover and in the Contents the name of the co-author, J. B. Appel, was unfortunately omitted. 